CERN-TH.6379/92

January, 1992

\title{
PERTURBING THE GROUND RING OF 2-D STRING THEORY
}

\author{
J.L.F. BARBÓN \\ Theory Division, CERN \\ CH-1211 Geneva 23, Switzerland \\ and \\ Departamento de Física Teórica \\ Universidad Autónoma de Madrid \\ Cantoblanco 28049, Madrid $^{\star}$
}

\begin{abstract}
We use free field techniques in $\mathrm{D}=2$ string theory to calculate the perturbation of the special state algebras when the cosmological constant is turned on. In particular, we find that the "ground cone" preserved by the ring structure is promoted to a three dimensional hyperboloid as conjectured by Witten. On the other hand, the perturbed $(1,1)$ current algebra of moduli deformations is computed completely, and no simple geometrical interpretation is found. We also quote some facts concerning the Liouville/ matrix model dictionary in this class of theories.
\end{abstract}

\footnotetext{
$\star$ Permanent address
} 


\section{Introduction}

Many efforts have been focused in recent times on the study of $\mathrm{c}=1$ non critical strings, $\mathrm{D}=2$ critical strings in disguise. They are interesting as toy models for the study of the classical open problems in string theory, like background dynamics, string field theory and other non perturbative methods. The matrix model formulation is extremely powerful in actual calculations, and allows for a complete solution of the model to all orders in perturbation theory, and even more than that, it provides a framework in which one can study non perturbative effects. Despite the successes of matrix models as calculational tools, we still need the usual continuum formulation in order to settle the physical picture, like the spectrum and the role of the symmetries.

Naively one could expect decoupling of two chains of string oscillators, just as in the critical 26 dimensional string, leaving only the center of mass degree of freedom (the tachyon, which is massless in $\mathrm{D}=2$ ). However, a careful analysis shows the existence of an infinite discrete set of massive oscillator degrees of freedom which fail to decouple. These are the so called singular states, first identified in the matrix model [1] and resonant Liouville correlators [2], and subsequently studied in full detail by the BRST procedure [3] . Choosing an appropiate gauge one can write them as a singular vector in $\mathrm{c}=1$ Virasoro times the standard gravitational dressing of Liouville:

$$
W_{s, n}^{ \pm}=V_{s, n} e^{\sqrt{2}(1 \mp s) \phi}
$$

where $V_{s, n}$ can be computed most easily using the SU(2) Kac-Moody algebra at $\mathrm{c}=1$,

$$
V_{s, n} \sim\left(\oint e^{-i \sqrt{2} \psi}\right)^{s-n} e^{i \sqrt{2} s \psi}
$$

for $s=0,1 / 2,1, \ldots ;|n|<s$, the $S U(2)$ indices, and $\psi$ denotes the (non-compact)

$c=1$ free boson. For the compact case we still have this $S U(2)$ structure at radius $R=1 / \sqrt{2}$ and $s=$ integer. 
Remarkably enough, this currents enlarge the symmetry of the theory to $W_{1+\infty}$ [4]. [5] [6]

$$
\left[W_{s, n}, W_{s^{\prime}, n^{\prime}}\right]=\left(s n^{\prime}-s^{\prime} n\right) W_{s+s^{\prime}-1, n+n^{\prime}}
$$

The role of the singular states is fully clarified in the beautiful analysis by Witten [4], who found that the exotic (non standard ghost number) operators in the BRST cohomology generate a ring structure under OPE. The previously found special currents act on this operator ring as area preserving vector fields, that is, the ground ring provides a representation space for $W_{1+\infty}$. The overall picture is the following: for the free case (no cosmological term in the action) the so called ground ring of ghost number 0 , spin $(0,0)$ operators has four generators, $a_{i} i=1,2,3,4$, satisfying the condition $a_{1} a_{2}-a_{3} a_{4}=0$, which defines a 3 dimensional cone in $a_{i}$ space. Out of the $W$ currents and the $a_{i}$ we can construct $(1,0)$ and $(0,1)$ (non chiral) currents $J_{s, n, n^{\prime}}(z, \bar{z})\left(n=n^{\prime}\right.$ for the non compact case) realizing an algebra of area preserving vector fields on the ground cone. In fact, $a_{1}$ and $a_{2}$ correspond to the coordinates of the fermionic phase space in the matrix model formulation, while $\partial \psi-\overline{\partial \psi}$, the operator measuring the left-right momentum difference, corresponds to the time coordinate in the matrix model. In the uncompactified case, all operators should be invariant under its action, and the previous structure collapses to the area preserving diffeomorphisms of the $a_{1}, a_{2}$ plane $\left(W_{1+\infty}\right)$ that also preserve the locus $a_{1} a_{2}=0$, which in turn is related to the Fermi surface of the matrix model.

It was conjectured in [4] that the non zero cosmological constant correction to this structure amounts simply to change the cone into a more general quadric, $a_{1} a_{2}-a_{3} a_{4}=\mu$, and the Fermi surface into $a_{1} a_{2}=\mu$. In this case the currents $J, \bar{J}$ would act as volume preserving vector fields of this quadric.

On the other hand, $(1,1)$ currents constructed as left-right producs of W's,

$$
Z_{s, n, n^{\prime}}^{ \pm}=W_{s, n}^{ \pm} \bar{W}_{s, n^{\prime}}^{ \pm}
$$

( $n=n^{\prime}$ for the non compact model) correspond to the infinitesimal moduli related 
to marginal deformations of the free $\mathrm{D}=2$ string. Their current algebra is $\left(W_{1+\infty}\right)^{2}$ for the free case, and the structure constants determine the beta functions to second order in the couplings. It was conjectured in [5] that for $\mu \neq 0$ this $W_{1+\infty}$ deforms into some $S L(2, R)$ tensor operator algebra $\mathcal{T}(\lambda)(\lambda=$ casimir $)$. In particular $\mathcal{T}(\infty)$ is the algebra of area preserving diffeomorphisms of a two dimensional hyperboloid $[7]$.

In the present work, we try to get some insight on this conjectures. In particular, we certainly find a deformation of both the Fermi surface and the ground cone, while a geometrical interpretation of the moduli current algebra is still elusive, in spite of the fact that the complete structure constants present a relatively simple pattern, after cancelation by the Liouville sector of most of the tachyon-like terms. Also, an interpretation is given of what the perturbed algebra of the $J, \bar{J}$ currents would be. Unless otherwise stated, we consider the non-compact model in concrete computations.

\section{Perturbation of the ground ring}

Let's first fix our notation. $X=(\psi, \phi)$ denotes the matter/Liouville coordinates and the total e.m. tensor is

$$
T_{t o t}(z)=-2 b(z) \partial c(z)-c(z) \partial b(z)-\frac{1}{2} \partial X_{\mu} \partial X^{\mu}(z)+\frac{i \tilde{Q}^{\mu}}{2} \partial^{2} X_{\mu}
$$

where $\tilde{Q}=\left(\alpha_{0},-i Q\right)$ is the background charge vector, given by $(0,-2 \sqrt{2} i)$ for $c=1$. We assume the euclidean metric and consider imaginary momenta in the Liouville direction, $p=(k,-i \beta)$.

Physical $(0,0)$ ghost number 0 chiral operators have the following "tachyon + photon" form

$$
x_{ \pm}(z)=\mathcal{O}_{\frac{1}{2}, \pm \frac{1}{2}}(z)=\left(j(z)+i \xi_{\mu}^{ \pm} \cdot \partial X^{\mu}(z)\right) e^{i p^{ \pm} \cdot X(z)}
$$

$j(z)=c(z) b(z)$ is the standard ghost current and the allowed momenta/polarizations are given by $p^{ \pm}=( \pm 1 / \sqrt{2}, i / \sqrt{2}), \xi^{ \pm}=( \pm 1 / \sqrt{2},-i / \sqrt{2})$ 
for the special states at $c=1$. We can combine this basic chiral fields in four ways to form full quantum operators:

$$
a_{+}=a_{1}=x_{+} \bar{x}_{+} ; a_{-}=a_{2}=x_{-} \bar{x}_{-} \quad ; \quad a_{3}=x_{+} \bar{x}_{-} ; a_{4}=x_{-} \bar{x}_{+}
$$

and the quadratic relation defining the cone $a_{1} a_{2}-a_{3} a_{4}=0$ arises automatically because of chiral factorization, (in the non-compact model we must balance matter momenta between $a_{3}$ and $a_{4}$ ). However, in a fully interacting Liouville sector we do not expect this operators to have a simple chiral square root, allowing for Witten's hyperbolic deformation $a_{1} a_{2}-a_{3} a_{4}=\mu$. One should understand this equation as valid in correlation functions or under the perturbed OPE of currents acting on $a_{i}$ polynomial functions defined on the quadric. Indeed, we'll explicitly calculate the normalized correlator

$$
\frac{1}{Z(\mu)}\left\langle\left(a_{1} a_{2}-a_{3} a_{4}\right)\right\rangle_{\mu} \sim \mu
$$

with an appropiate renormalization of the $a_{i}$. To evaluate (2.4) when we add $\mu$ requires a suitable definition of the product involved i.e. a regularization. At this point we can take advantage of the fact that the $a_{i}$ operators enjoy a ring structure with a singularity free OPE, at least up to BRST trivial operators. Then a natural regularization would be just point splitting.

$$
\begin{gathered}
\lim _{\epsilon_{ \pm} \rightarrow 0}\left(a_{1}\left(\epsilon_{+}\right) a_{2}\left(-\epsilon_{-}\right)-a_{3}\left(\epsilon_{+}\right) a_{4}\left(-\epsilon_{-}\right)\right)= \\
\lim _{\epsilon_{ \pm} \rightarrow 0} x_{+}\left(\epsilon_{+}\right) x_{-}\left(-\epsilon_{-}\right)\left(\bar{x}_{+}\left(\bar{\epsilon}_{+}\right) \bar{x}_{-}\left(-\bar{\epsilon}_{-}\right)-\bar{x}_{-}\left(\bar{\epsilon}_{+}\right) \bar{x}_{+}\left(-\bar{\epsilon}_{-}\right)\right)
\end{gathered}
$$

Remarkably enough, this limit is non zero when we allow the $a_{i}$ to interact with the cosmological constant operator.

Before addressing this point, let's consider some general properties of correlation functions of $a_{i}$ operators. To be specific, we look at correlators of the form 
$\left(Q=\frac{2}{\gamma}+\gamma\right)$

$$
\frac{\mu^{s} \Gamma(-s)}{Z(\mu)}\left\langle a_{+}\left(\epsilon_{+}\right) a_{-}\left(\epsilon_{-}\right)\left(\int e^{\gamma \phi}\right)^{s} \prod_{j=1}^{N} \int T_{p_{j}}\right\rangle_{\mu=0}
$$

where we have integrated out the Liouville zero mode in the standard way, and introduced $\mathrm{N}$ tachyons $T_{p_{j}}=e^{i p_{j} \cdot X}, p_{j}=\left(k_{j},-i \beta_{j}\right)$ for generality. We consider only "bulk" correlators, for which $s=\frac{1}{\gamma}\left(Q-\beta_{+}-\beta_{-}-\sum \beta_{j}\right)$ is a positive integer. In that case we interpret the infinite in $\Gamma(-s)$ as a volume divergence associated with the Liouville coordinate, it is natural then to susbstitute $\Gamma(-s) \rightarrow(-)^{s}|\log \mu| / s !$. Taking into account the scaling of the $\mathrm{c}=1$ partition function, $Z(\mu) \sim \mu^{2}|\log \mu|$ we can write the correlator of interest in (2.6) as

$$
F\left(\epsilon_{+}, \epsilon_{-} ; \mu\right)=(-)^{s} \frac{\mu^{s-2}}{s !} \int \prod_{i=1}^{s} d^{2} w_{i} \prod_{j=1}^{N} d^{2} z_{j} \mathcal{A} \overline{\mathcal{A}}
$$

the unintegrated chiral amplitude is given by

$$
\mathcal{A}\left(\epsilon_{+},-\epsilon_{-}, w_{i}, z_{j}\right) \equiv \mathcal{A}\left(Z_{\alpha}\right)=\left\langle x_{+}\left(\epsilon_{+}\right) x_{-}\left(-\epsilon_{-}\right) \prod_{\alpha \neq \pm} e^{i P_{\alpha} \cdot X\left(Z_{\alpha}\right)}\right\rangle
$$

where $P_{\alpha}=(p_{+}, p_{-}, \overbrace{p_{\gamma}, \ldots, p_{\gamma}}^{s}, p_{j}), \quad p_{\gamma}=(0,-i \gamma)$.

The resulting free $\mu=0$ correlators are performed using free field contractions based on the propagator

$$
\left\langle X^{\mu}(z) X^{\nu}(w)\right\rangle=-\delta^{\mu \nu} \log (z-w)
$$

The "photon" terms are treated in the standard way in critical string amplitudes, and the integrals above should be understood as continued from $c<1$. In this 
way, using the cosmological constant operator

$$
\mu \tilde{T}_{\gamma}=\mu \frac{e^{\gamma \phi}}{\Delta(-\rho)} ; \quad \Delta(x) \equiv \frac{\Gamma(x)}{\Gamma(1-x)} ; \quad \rho \equiv-\frac{\gamma^{2}}{2}
$$

in the $c \rightarrow 1$ limit we effectively insert the true microscopic operator (in the terminology of [8] $\mu \phi e^{\gamma \phi}$ ).In order to actually calculate the integrals we must tune the momenta $p_{j}, p_{ \pm}$in such a way that charge conservation is achieved

$$
p_{+}+p_{-}+s p_{\gamma}+\sum_{j=1}^{N} p_{j}=\tilde{Q}
$$

with s a positive integer. For $N=0$, this conditions plus the $(1,1)$-operator conditions uniquely fix the momenta, for given $\alpha_{0}$ and $s$. Thus in general there is not enough freedom to tune the singular operator's momenta. Although this operators only exist as BRST non trivial classes for particular momenta, they are generically Virasoro primaries, in contrast with the case of $(1,1)$ discrete currents. This is easily seen by performing the OPE of $x_{ \pm}$with the total e.m. tensor (2.1) . They are $(0,0)$ Virasoro primaries if

$$
L_{1} x_{ \pm}=\left(\xi^{ \pm} \cdot\left(p^{ \pm}-\tilde{Q}\right)-3\right) x_{ \pm}=0 \quad L_{0} x_{ \pm}=\left(\frac{1}{2} p^{ \pm} \cdot\left(p^{ \pm}-\tilde{Q}\right)+1\right) x_{ \pm}=0
$$

where the factor of 3 comes from the ghost anomaly on the sphere. The important point is that these are non-homogeneus equations in $p$ and $\xi$, thus having generically non-trivial solutions. This is an ilustration of the fact that BRST cohomology is rather subtle for those operators with ghost content. In particular, the $x_{ \pm}$'s have an associated current $U_{ \pm}(z)=b_{-1}\left(x_{ \pm}\right)=b(z) \exp \left(i p^{ \pm} \cdot X(z)\right)$, but by fixing again this current in the standard way, we obtain $c(z) U_{ \pm}(z)=j(z) \exp \left(i p^{ \pm} \cdot X(z)\right)$. It seems that we have lost the "photon" part. In fact, the 1-1 correspondence between BRST closed states and (0,0) Virasoro primaries occurs only for ghost free fixed currents of the form $c(z) \times(1,1) \operatorname{current}(\psi, \phi)$ as for critical strings.

The net outcome of this disgression is that we can tune the polarizations $\xi$ in order to have Virasoro primary operators along our $\mathrm{s}=$ integer trajectory $c \rightarrow 1^{-}$. 


\subsection{Fermi SURFACE}

The KPZ scaling of the product $a_{+} a_{-}$is $\mu^{3}$. This means that $a_{+} a_{-} \sim \mu$ in normalized correlators like (2.6) in agreement with the expectations in [4]. Of course we must show that the proportionality constant (the integral in (2.7) ) is non vanishing.

Let's calculate the chiral amplitude $\mathcal{A}$ in (2.8). We may split it in two terms $\mathcal{A}_{0}+\mathcal{A}_{1}$ : a "tachyon" part,

$$
\begin{gathered}
\mathcal{A}_{0}=\left\langle j\left(\epsilon_{+}\right) j\left(-\epsilon_{-}\right)\right\rangle\left\langle\prod_{\alpha} e^{i P_{\alpha} \cdot X\left(Z_{\alpha}\right)}\right\rangle= \\
=\frac{1}{\left(\epsilon_{+}+\epsilon_{-}\right)^{2}} \prod_{\alpha<\beta} Z_{\alpha \beta}^{P_{\alpha} \cdot P_{\beta}} ; \quad\left(Z_{\alpha \beta}=Z_{\alpha}-Z_{\beta}\right)
\end{gathered}
$$

and a "photon" part,

$$
\begin{gathered}
\mathcal{A}_{1}=\left\langle i \xi^{+} \cdot \partial X e^{i p^{+} \cdot X\left(\epsilon_{+}\right)} i \xi^{-} \cdot \partial X e^{i p^{-} \cdot X\left(-\epsilon_{-}\right)} \prod_{\alpha \neq \pm} e^{i P_{\alpha} \cdot X\left(Z_{\alpha}\right)}\right\rangle= \\
=\left\{\frac{\xi^{+} \cdot \xi^{-}}{\left(\epsilon_{+}+\epsilon_{-}\right)^{2}}+\left(f^{+} \cdot \xi^{+}\right)\left(f^{-} \cdot \xi^{-}\right)\right\} \prod_{\alpha<\beta} Z_{\alpha \beta}^{P_{\alpha} \cdot P_{\beta}}
\end{gathered}
$$

where

$$
f^{ \pm}=\frac{ \pm p_{\mp}}{\epsilon_{+}+\epsilon_{-}}+\sum_{k=1}^{s} \frac{p_{\gamma}}{ \pm \epsilon_{ \pm}-w_{k}}+\sum_{j=1}^{N} \frac{p_{j}}{ \pm \epsilon_{ \pm}-z_{j}}
$$

Taking into account that $\xi^{+} \cdot \xi^{-} \rightarrow-1$ and $\xi^{ \pm} \cdot p^{\mp} \rightarrow 0$ as $c \rightarrow 1$, our final result, up to terms vanishing in this limit, is

$$
\begin{gathered}
\mathcal{A}\left(Z_{\alpha}\right)=\left(h^{+} \cdot \xi^{+}\right)\left(h^{-} \cdot \xi^{-}\right) \prod_{\alpha<\beta}\left|Z_{\alpha \beta}\right|^{P_{\alpha} \cdot P_{\beta}} \\
h^{ \pm}=\sum_{\alpha \neq \pm} \frac{P_{\alpha}}{ \pm \epsilon_{ \pm}-Z_{\alpha}}
\end{gathered}
$$

After changing variables $Z_{\alpha} \rightarrow\left(\epsilon_{+}-Z_{\alpha}\right) /\left(\epsilon_{+}+\epsilon_{-}\right)$in the integral (2.8), and making 
repeated use of the on shell conditions, (charge conservation and conformal weights 0 and 1 for fixed and moving operators resp.), we find that the dependence in $\epsilon_{ \pm}$ cancels as it should be, since we are dealing with the two point function of $(0,0)$ operators. In fact, for the particular case in which all $P_{\alpha}$ are equal to each other $\left(P_{\alpha}=p_{\gamma}\right)$ the problem is simply reduced to a tachyon amplitude, because in this case we can trade the $h^{ \pm}$terms by a differential operator

$$
\mathcal{A}\left(Z_{\alpha}\right)=-\left(\epsilon_{+}+\epsilon_{-}\right)^{p^{+} \cdot p^{-}} \frac{\xi^{+} \cdot p_{\gamma}}{\xi^{+} \cdot p_{\gamma}} \partial_{\epsilon_{+}} \partial_{\epsilon_{-}} \prod_{\alpha \neq \pm}\left(\epsilon_{+}-Z_{\alpha}\right)^{p^{+} \cdot p_{\gamma}}\left(-\epsilon_{-}-Z_{\alpha}\right)^{p^{-} \cdot p_{\gamma}} \prod_{\alpha<\beta \neq \pm} Z_{\alpha \beta}^{P_{\alpha} \cdot P_{\beta}}
$$

Inserting this expression in (2.7) we end up with the by now famous B-9 integral of [9], $J(\alpha, \beta, \rho)$. In our case $\alpha=\beta \rightarrow 1$ and $\rho=-\gamma^{2} / 2 \rightarrow-1$. Using the Liouville charge relation we have, $\alpha=-1-\rho(N+s-1)$ and after some algebra we get

$$
J_{s+N}(\alpha, \alpha, \rho) \sim \Delta(0) \prod_{i=1}^{s+N-1} \Delta(-i \rho)
$$

We find a divergent result!. In fact, this divergence along all the $c \rightarrow 1^{-}$trajectory is due to the volume of the dilatation group, which remains from $S L(2, C)$ after fixing two points. In order to divide out this volume we can factor the Haar measure for dilatations,

$$
\int d^{2} \gamma \int d^{2} \beta \frac{d^{2} \alpha \delta^{2}(\gamma) \delta^{2}(\beta-1)}{|\alpha-\beta|^{2}|\alpha-\gamma|^{2}|\beta-\gamma|^{2}}=\frac{d^{2} \alpha}{|\alpha|^{2}|1-\alpha|^{2}}
$$

and fix, say $\alpha=\infty$. The net result is just the elimination of one of the integrals:

$$
\frac{J_{s+N}}{\operatorname{Vol}(\operatorname{dil})}=J_{s+N-1}(\alpha, \alpha, \rho) \sim \Delta(-\rho)^{N} \Delta(1+(s+N) \rho)
$$

Upon using once more the Liouville charge relation we have $N+s=3$. If we also renormalize the new $\mathrm{N}$ tachyons by the usual leg factor $\Delta(-\rho)^{-1}$, and the 
product $a_{+} a_{-}$by $\Delta(-3 \rho)$ we finaly arrive at

$$
\frac{1}{Z(\mu)}\left\langle a_{+} a_{-} \tilde{T}_{\gamma}^{N}\right\rangle_{\mu} \sim \frac{(-)^{N}}{(3-N) !} \mu^{1-N}
$$

The renormalization of the $a_{ \pm}$operators is divergent in the $c=1$ limit. Maybe it could be interpreted as a kind of "leg pole" similar to the ones occurring in the special tachyon correlators. In consecuence we see that the properly renormalized product $a_{+} a_{-} \equiv a_{1} a_{2}$ is proportional to $\mu$ inside correlators of cosmological constant operators. Note however that the proportionality constant in (2.21) depends on the number $\mathrm{N}$ of tachyons. This could be due to the effect of non-decoupled BRST conmutators. After all, as was observed in [10], the cosmological constant is in the same module (representation) of the ring as the more complicated discrete states. In this module the free relation $a_{+} a_{-}=0$ is not supposed to be valid. However, since

$$
\left\langle\tilde{T}_{\gamma}^{N+1}\right\rangle_{\mu} \sim\left(\frac{\partial}{\partial \mu}\right)^{N-2} \mu^{s+N-2} \sim \frac{\mu^{3-N}}{(3-N) !}
$$

we find the following operator identification inside correlators of cosmological constants

$$
a_{1} a_{2} \sim \mu \tilde{T}_{\gamma}
$$

\subsection{Ground CONE}

Now we are ready to check the deformation of the ground cone. According to (2.5) we must calculate

$$
C(\mu)=\lim _{\epsilon_{ \pm} \rightarrow 0} \frac{1}{Z(\mu)}\left\langle\left(a_{1}\left(\epsilon_{+}\right) a_{2}\left(-\epsilon_{-}\right)-a_{3}\left(\epsilon_{+}\right) a_{4}\left(-\epsilon_{-}\right)\right) \prod_{j} \int T_{p_{j}}\right\rangle_{\mu}
$$

In terms of the chiral amplitudes introduced before,

$$
C(\mu) \sim \mu^{1-N} \int \prod_{i=1}^{s} d^{2} w_{i} \prod_{j=1}^{N} d^{2} z_{j} \mathcal{A}\left(\epsilon_{+},-\epsilon_{-}\right)\left(\overline{\mathcal{A}}\left(\bar{\epsilon}_{+},-\bar{\epsilon}_{-}\right)-\overline{\mathcal{A}}\left(-\bar{\epsilon}_{-}, \bar{\epsilon}_{+}\right)\right)
$$

and all we need is to compute the braiding between the two special operators in 
the antiholomorphic part of the unintegrated correlator. In the general case there is no simple expression for this braiding. But again for the case $P_{\alpha}=p_{\gamma}$ we find a big simplification. The corresponding chiral amplitude is given by (2.17), whose braiding phase is $(-)^{p_{+} \cdot p_{-}}$, just -1 at $c=1$. In consecuence, $(2.24)$ is exactly twice the integrals in the previous section.

Such a result might seem paradoxical since we already argued that the final amplitudes are independent of $\epsilon_{ \pm}$. However, note that the braiding is taken before integrating the moving operators, this is technically the explanation of why pointsplitting actually works.

To conclude, we have shown that, assuming the same renormalizations as in the previous section

$$
\frac{1}{Z(\mu)}\left\langle\left(a_{1} a_{2}-a_{3} a_{4}\right) \tilde{T}_{\gamma}^{N}\right\rangle_{\mu} \sim \mu^{1-N}
$$

So, at least in the presence of the cosmological constant operator, we have the identification

$$
a_{1} a_{2}-a_{3} a_{4} \sim \mu \tilde{T}_{\gamma}
$$

\section{Perturbed current algebra of moduli deformations}

Generally speaking, $(1,1)$ currents are associated to marginal deformations of the theory, at least on a first approximation. Trully marginal deformations, (so called integrably marginal) correspond to $(1,1)$ currents to all orders in perturbation theory. In the uncompactified model, the moduli associated with infinitesimal deformations of the 2-D free string are

$$
Z_{s, n}^{ \pm}=W_{s, n}^{ \pm} \bar{W}_{s, n}^{ \pm}
$$


For a general perturbation of the form

$$
S \rightarrow S+\sum_{a} \lambda_{a} \int Z_{a}
$$

their current algebra structure constants $K_{a, b}^{c}$ determine the beta functions up to second order in the couplings,

$$
\beta^{c} \sim \sum_{a, b} \lambda_{a} \lambda_{b} K_{a, b}^{c}
$$

In was found in [4] and [5] that this structure constants correspond to $W_{1+\infty}^{2}$ at the free point $\mu=0$. In fact, it is very easy to see that perturbation theory in $\lambda_{a}$ is exact for any simple deformation of the free lagrangian involving only one $Z_{s, n}^{ \pm}$operator. This is due to the fact that this $Z$ operators carry definite 2-D momentum, so that the perturbative expansion of any correlator is truncated to a single term in the same way as in the lagrangian formulation of Coulomb gas conformal field theories (see [9]). For more general linear combinations of the Z's this is no longer true, just like the symmetric perturbation of screenings in the Coulomb gas gives SineGordon theory, which is a massive theory (of course this remarks only apply in a perturbative sense; exact evaluation of the zero mode à la Goulian-Li drastically changes the situation). Looking at the perturbed two point function of $\mathrm{Z}$ operators, one can easily see that the only non trivial (single) perturbation is $Z_{1,0}^{+}=\partial \psi \overline{\partial \psi}$ which corresponds to the well known radial moduli already present in the $\mathrm{c}=1$ matter theory. In the general case it is much more difficult to decide whether a particular perturbation is marginal or not. For example, the second order beta function in (3.3) vanishes for perturbations in abelian subalgebras of $W_{1+\infty}^{2}$. Taking for simplicity deformations by "microscopic" $(+)$ operators, and considering the non compact case, we have the general second order marginal deformation

$$
\mathcal{D}_{s, n}=\sum_{s^{\prime}} \lambda_{s^{\prime}} Z_{s^{\prime}, n \frac{s^{\prime}}{s}}^{+}
$$

(see [4] for an expression in terms of $a_{i}$ operators). 
Our aim in this section is to calculate the deformation of the structure constants along the marginal line corresponding to the pure cosmological constant perturbation. This is equivalent to the computation of three point functions of $Z$ operators $\left\langle Z^{+} Z^{+} Z^{-}\right\rangle$from which we can read off the $K_{++}^{+}$and the $K_{+-}^{+}$structure constants. It was already shown in [11] the vanishing of all $\left\langle Z^{+} Z^{+} Z^{+}\right\rangle$correlators. So we just consider the $K_{++}^{+}, K_{--}^{+}, K_{--}^{-}$cases.

The chiral vertex operators are constructed as in (1.1) where we normalize the matter operators by the Condon-Shortley convention,

$$
V_{s, n}=\left[\frac{(s+n) !}{(s-n) !(2 s) !}\right]^{1 / 2}\left(\oint J_{-}\right)^{s-n} V_{s, s}
$$

$V_{s, s}$ is just the usual vertex operator $\exp (i \sqrt{2} s \psi), \quad s \in \mathbf{Z} / 2$ and $J_{ \pm}=$ $\exp \{ \pm i \sqrt{2} \psi\}, \quad J_{3}=i \sqrt{2} \partial \psi$ satisfy the $S U(2)$ Kac-Moody current algebra at level $k=1$. In this way $V_{s, n}$ is proportional to a polynomial in derivatives of the $\psi$ field, times the vertex $\exp \{i n \sqrt{2} \psi\}$ carrying the momentum. The total two dimensional momentum of the $W_{s, n}^{ \pm}$fields is $p=(k,-i \beta)=\sqrt{2}(n,-i(1 \mp s))$.

We are interested in correlators of the form $K_{++}^{+} \sim<Z_{s_{1}, n_{1}}^{+} Z_{s_{2}, n_{2}}^{+} Z_{s_{3},-n_{3}}^{-}>_{\mu}$. After $S L(2, C)$ fixing and zero mode integration we have

$$
K_{s_{1}, n_{1} ; s_{2}, n_{2}}^{s_{3}, n_{3}}=(-)^{s} \frac{\mu^{s}}{s !}|\log \mu|\left\langle Z_{s_{1}, n_{1}}^{+}(0) Z_{s_{2}, n_{2}}^{+}(1) Z_{s_{3},-n_{3}}^{-}(\infty)\left(\int \tilde{T}_{\gamma}\right)^{s}\right\rangle_{\mu=0}
$$

for $\tilde{T}_{\gamma}$ the renormalized cosmological constant operator as in (2.10) . In the sequel, we will drop the Liouville volume term $|\log \mu|$ when speaking about the current algebra structure constants. Since $s$ is always integer for correlators of singular operators, it is consistent to integrate the Liouville zero mode along the imaginary axis. In this way we make contact with the free $(\mu=0)$ case.

We can split (3.6) into matter and Liouville parts

$$
K_{s_{1}, n_{1} ; s_{2}, n_{2}}^{s_{3}, n_{3}}=\frac{\mu^{s}}{s !}(-)^{s}\left(f_{s_{1} s_{2}}^{s_{3}} C_{n_{1} n_{2} ; n_{3}}^{s_{1} s_{2} ; s_{3}}\right)^{2} L_{s_{1} s_{2}}^{s_{3}}
$$

where $L$ is the Liouville part, $C$ is the usual Clebsch-Gordan coefficient, and $f_{s_{1} s_{2}}^{s_{3}}$ 
is the reduced matrix element in the matter OPE:

$$
V_{s_{1}, n_{1}}(z) \cdot V_{s_{2}, n_{2}}(0) \sim \sum_{s_{3}=\left|s_{1}-s_{2}\right|}^{s_{1}+s_{2}} z^{\Delta_{3}-\Delta_{1}-\Delta_{2}} f_{s_{1} s_{2}}^{s_{3}} C_{n_{1}, n_{2} ; n_{3}}^{s_{1}, s_{2} ; s_{3}} V_{s_{3}, n_{3}}(0)+\ldots
$$

The kinematics in (3.6) enforces the selection rules: $n_{3}=n_{1}+n_{2} ; s=s_{1}+s_{2}-$ $s_{3}-1=$ positive integer, which combine with the Clebsch-Gordan rule to give $s_{3}=s_{1}+s_{2}-1, \ldots,\left|s_{1}-s_{2}\right|$. In particular, we learn from this constraints that $K_{--}^{ \pm}=0$ also for $\mu \neq 0$. Let's explicitly calculate the structure constants in (3.7).

\subsection{MATter StruCture CONStAntS}

We want to calculate the function $f_{s_{1} s_{2}}^{s_{3}}$. For the free case we have $s=0,\left(s_{3}=\right.$ $\left.s_{1}+s_{2}-1\right)$ and the result quoted in [5] is

$$
f_{s_{1} s_{2}}^{s_{3}}=-\frac{\left(2 s_{1}+2 s_{2}-2\right) !}{\left(2 s_{1}-1\right) !\left(2 s_{2}-1\right) !}
$$

Here we follow a similar argument. Consider the OPE $V_{s_{1}, s_{3}-s_{2}}(z) \cdot V_{s_{2}, s_{2}}(0)$ and identify the tachyon on the right hand side. That is, we select the term

$$
f_{s_{1} s_{2}}^{s_{3}} C_{s_{2}-s_{3}, s_{2} ; s_{3}}^{s_{1}, s_{2} ; s_{3}} V_{s_{3}, s_{3}}(0)
$$

which corresponds to the leading one in the following OPE

$$
\sqrt{\frac{\left(s_{1}+s_{3}-s_{2}\right) !}{\left(s_{1}+s_{2}-s_{3}\right) !\left(2 s_{1}\right) !}} \prod_{j=1}^{s+1} \oint \frac{d u_{j}}{2 \pi i}: e^{-i \sqrt{2} \psi\left(z+u_{j}\right)}:: e^{i s_{1} \sqrt{2} \psi(z)}:: e^{i s_{2} \sqrt{2} \psi(0)}:
$$

After performing the contractions and writing explicitly the Clebsch-Gordan coefficient (fortunately this can be done in this case, see [12]), we find

$$
f_{s_{1} s_{2}}^{s_{3}}=\left[\frac{\left(s_{1}+s_{2}-s_{3}\right) !\left(s_{2}+s_{3}-s_{1}\right) !\left(s_{1}+s_{2}+s_{3}-1\right) !}{\left(2 s_{1}\right) !\left(2 s_{2}\right) !\left(s_{1}+s_{2}-s_{3}\right) !\left(2 s_{1}+1\right) !}\right]^{1 / 2} I\left(s_{1}, s_{2}, s_{3}\right)
$$


and $I\left(s_{1}, s_{2}, s_{3}\right)$ denotes the integral

$$
I\left(s_{1}, s_{2}, s_{3}\right)=\prod_{j=1}^{s+1} \oint \frac{d x_{j}}{2 \pi i} x_{j}^{-2 s_{1}}\left(1+x_{j}\right)^{-2 s_{2}} \prod_{j<k}^{s+1}\left(x_{j}-x_{k}\right)^{2}
$$

This integral is explicitly computed in the Appendix.

\subsection{Liouville Structure CONSTANTS}

The functions $L_{s_{1}, s_{2}}^{s_{3}}$ in (3.7) have already been computed in [11]. Here we will just comment on some delicate points.

The integral representation for the L's, prior to $S L(2, C)$ fixing is given by:

$$
\frac{1}{\operatorname{Vol}(S L(2, C))} \int \prod_{k=1}^{3} d^{2} z_{k} \prod_{i=1}^{s} d^{2} w_{i} \prod_{k<l}^{3}\left|z_{k l}\right|^{2 \delta_{k l}} \prod_{k, i}\left|w_{i}-z_{k}\right|^{-2 \gamma \beta_{k}} \prod_{i<j}^{s}\left|w_{i j}\right|^{4 \rho}
$$

Naive substitution of the $c=1$ exponents gives a divergent integral and the appropiate regularization is not known from the Liouville point of view. The reason is that, unlike the case of the $a_{i}$ operators in the previous section, there are no $(1,1)$-discrete currents in $c<1$. So, the best one can do at this point is to continue the exponents in (3.14), while maintaining at least $S L(2, C)$ invariance, leaving the matter structure constants untouched.

It is very easy to show that (3.14) is independent of the fixing of $z_{k}$ if we have Liouville-charge matching:

$$
\sum_{k} \beta_{k}+\gamma s=Q
$$

and the following three equations are satisfied:

$$
\sum_{l \neq k} \delta_{l k}=\gamma s \beta_{k}-2
$$

So, for fixed s and $\mathrm{Q}$, all $\delta_{k l}$ are determined by two of the $\beta_{k}$, say, $\beta_{0}$ and $\beta_{1}$. And we are left with the B-9 integral $J_{s}(\alpha, \beta, \rho)$, where $\alpha=-\gamma \beta_{0}, \quad \beta=-\gamma \beta_{1}, \quad \rho=-\gamma^{2} / 2$. 
The most general " $c<1$ " (projective invariant) regularized trajectory is thus given by

$$
\rho=\epsilon-1 ; \alpha=a \epsilon+2 s_{1}-2 ; \beta=b \epsilon+2 s_{2}-2 ; \epsilon \rightarrow 0
$$

and $a, b$ are free parameters. The choice made in [11] corresponds to the particular case $a=\frac{4}{3}-s_{1}$ and $b=\frac{4}{3}-s_{2}$. Note that we are changing the conformal properties of the matter part for all of this trajectories. To see this, recall that the $\delta_{k l}$ coefficients have the form

$$
\delta_{k l}=-\Delta_{k l}-\beta_{k} \beta_{l} ; \Delta_{i j} \equiv s_{i}^{2}+s_{j}^{2}-s_{k}^{2}, k \neq i, j
$$

If we ask the deformation in (3.17) to keep the matter weights $\Delta_{k l}$ unchanged, then equations $(3.15),(3.16)$ and (3.18) imply the trivial result: $s_{i}=0$.

Carrying on the standard manipulations and taking the limit in (3.17) one finds a remarkable result: the regularization dependent terms can be packed in

$$
S(a, b)=\prod_{i=0}^{s-1} \frac{(i+b)(i+a)}{(a+b+s-1+i)}=\frac{\Gamma(a+s) \Gamma(b+s) \Gamma(a+b+s-1)}{\Gamma(a) \Gamma(b) \Gamma(a+b+2 s-1)}
$$

while the "bulk" of the B-9 formula just gives the inverse of the squared integral $I\left(s_{1}, s_{2}, s_{3}\right)^{2}$ we found in the matter part (3.13). This is an interesting phenomenom, reminiscent of the similar one occurring for tachyon correlators. From this point of view, we find the cancelation of $\left(f_{s_{1}, s_{2}}^{s_{3}}\right)^{2}$ quite natural, since they are the $S U(2)$ reduced matrix elements in (3.8), thus depending on the special tachyons (i.e. the highest weights). 


\subsection{Complete structure COnstants}

If we collect the results in the previous sections and absorb some trivial factors in the normalization of the operators, we arrive at the following suggestive result for the complete structure constants

$$
K_{s_{1}, n_{1} ; s_{2}, n_{2}}^{s_{3}, n_{3}}=\mu^{s} \frac{\left(2 s_{3}\right)\left(2 s_{3}+1\right)\left(s_{1}+s_{2}+s_{3}+1\right)^{2}}{\left(s_{1}+s_{2}-s_{3}-1\right) !} S(a, b) \Delta\left(s_{1}, s_{2}, s_{3}\right)^{2}\left(C_{n_{1}, n_{2} ; n_{3}}^{s_{1}, s_{2} ; s_{3}}\right)^{2}
$$

where $\Delta\left(s_{1}, s_{2}, s_{3}\right)$ is Wigner's triangular function:

$$
\Delta(a, b, c)=\left[\frac{(a+b-c) !(a-b+c) !(b+c-a) !}{(a+b+c+1) !}\right]^{1 / 2}
$$

We still don't know what would be the geometrical interpretation of this structure constants (if any). The presence of the ambiguous term $S(a, b)$ complicates very much the analysis. In particular, they strongly resemble the structure constants of $\mathcal{T}(\infty)^{2}$ (see [7]), although no trace of the parity selection rule, $s_{1}+s_{2}+s_{3}=$ odd is seen. Besides, an interesting question about (3.20) is its matrix model interpretation. As pointed out in [13], in spite of the fact that left-right matching of momenta "explains" why only one $W_{\infty}$ is seen, at the level of correlation functions we still find a doubling of the group factors, since left and right parts of the $\mathrm{Z}$ operators contract independently. This puzzle is easily solved by realizing that, according to [4], the correct dictionary between the matrix model and the conformal theory is given by

$$
(p+\lambda)^{s+n}(p-\lambda)^{s-n} e^{2 m t} \leftrightarrow W_{s, n}^{+}(z) \overline{\mathcal{O}}_{s-1, n}(\bar{z}) \equiv J_{s, n}(z, \bar{z})
$$

for $p$ the momentum conjugate to the eigenvalue variable $\lambda$. Since the $\mathcal{O}^{\prime} s$ are in the ring, correlators of $J^{\prime} s$ do not double the group factors. However, this raises the question of how to write the $\mathrm{Z}$ moduli in terms of matrix model variables. 
Incidentally, we must say that a definite answer for (3.20) can be obtained from the operator solution in the Gervais-Neveu interacting Liouville theory [14] .In particular, one can construct the dressing operators explicitly in terms of chiral vertex operators lying in $S L(2, R)$ representations. This vertex operators enjoy the following remarkable OPE,

$$
e^{-\gamma s_{1} \phi(z, \bar{z})} \cdot e^{-\gamma s_{2} \phi(0)}=\sum_{s_{3}=\left|s_{2}-s_{1}\right|}^{s_{1}+s_{2}}|z|^{\Delta_{3}-\Delta_{2}-\Delta_{1}} e^{-\gamma s_{3} \phi(0)}+\ldots
$$

It is just the free one, but opened to all $S U(2)$ channels. So in this formulation the final result for the moduli OPE is given by

$$
K_{s_{1}, n_{1} ; s_{2}, n_{2}}^{s_{3}, n_{3}}=\mu^{s}\left(f_{s_{1} s_{2}}^{s_{3}} C_{n_{1} n_{2} ; n_{3}}^{s_{1} s_{2} ; s_{3}}\right)^{2} \quad ; \quad s_{3}=\left|s_{1}-s_{2}\right|+1, \ldots, s_{1}+s_{2}-1
$$

the only difference with the pure matter structure constants is the selection rule at the bottom. In would be interesting to understand why (3.20) and (3.24) look so different.

\section{Perturbation of $W_{1+\infty}$}

The study of the perturbed algebra of volume preserving diffeomorphisms along the same lines as in the previous section faces a fundamental problem. Recall the expression for the $(1,0)$ and $(0,1)$ currents,

$$
J_{s, n, n^{\prime}}(z, \bar{z})=W_{s, n}^{+}(z) \overline{\mathcal{O}}_{s-1, n^{\prime}}(\bar{z})
$$

and similarly for $\bar{J}$. $\left(\mathcal{O}_{s-1, n}\right.$ is BRST equivalent to $\left.x_{+}^{s+n-1} x_{-}^{s-n-1}\right)$. Their free OPE is just $W_{1+\infty} \oplus W_{1+\infty}$. However, we cannot conjugate $J$ and $\bar{J}$ in the Liouville momentum in order to get orthogonal two point functions. The reason being that the operators analogous to (4.1), but constructed out of the $W^{-}$currents, are unphysical. This is a striking feature of the free theory, which already distinguishes between $(+)$ and $(-)$ operators. In consecuence, we cannot establish a simple correspondence between OPE and 3-point functions. 
Indeed, from the matrix model point of view, nothing special happens when the cosmological constant is turned on (in fact, what is problematic in matrix models is the limit $\mu \rightarrow 0)$ ). The matrix model operators in (3.22) still satisty $W_{1+\infty}$ at $\mu \neq 0$.On the other hand, on general grounds, for non zero cosmological constant we don't expect the quantum operators to factorize in in chiral left/right terms in a simple way like in (4.1). As we'll see in a moment, this observation is important.

Let's consider the generalized quantum currents (4.1) for $\mu \neq 0$. According to the ansatz in [4] they are associated to a certain volume preserving vector field on the quadric $a_{1} a_{2}-a_{3} a_{4}=\mu$

$$
J_{s, n, n^{\prime}}(\mu) \leftrightarrow \mu^{s} w_{A_{1}, \ldots, A_{2 s} A_{2 s+1} A_{2 s+2} ; A_{1}^{\prime}, \ldots, A_{2 s}^{\prime}} x^{A_{1} A_{1}^{\prime}} \ldots x^{A_{2 s} A_{2 s}^{\prime}} x^{A_{2 s+1} B^{\prime}} \epsilon^{A_{2 s+2} B} \frac{\partial}{\partial x^{B B^{\prime}}}
$$

for AA' the $S U(2)$ indices for the four coordinates of the quadric. In the non compact case, we restrict (4.2) to those vector fields invariant under the operator computing the left-right momentum difference: $\partial \psi-\overline{\partial \psi} \leftrightarrow a_{3} \frac{\partial}{\partial a_{3}}-a_{4} \frac{\partial}{\partial a_{4}}$. Expanding the conmutator of two of these vector fields in the $S U(2) \times S U(2)$ Clebsch-Gordan series we arrive at the following fusion rules

$$
\left[J_{s_{1}}\right] \otimes\left[J_{s_{2}}\right] \sim \bigoplus_{s_{3}=\left|s_{2}-s_{1}\right|+1}^{s_{1}+s_{2}-1} \mu^{s_{1}+s_{2}-s_{3}-1}\left[J_{s_{3}}\right] ; \quad s_{1}+s_{2}+s_{3}=\text { odd }
$$

(the odd selection rule is due to parity conservation). This algebra contracts to $W_{1+\infty}$ as $\mu \rightarrow 0$. The puzzling question is that we never see the channels $s_{3}<$ $s_{1}+s_{2}-1$ in the matrix model. The answer becomes obvious as we descend from the homogeneus variables in (4.2) to the minimal set of variables $a_{1}, a_{2}, \psi$ for $a_{3}=\rho e^{\psi} a_{4}=\rho e^{-\psi}$. The set of diagonal vector fields in (4.2) is given by abelian gauge transformations in the $\psi$ direction, plus those area preserving vector fields of the $a_{1}-a_{2}$ plane that "lift" correctly to the homogeneus form in (4.2). That is, those generated by hamiltonians of the form:

$$
h\left(a_{1}, a_{2}\right)=\left(a_{1} a_{2}-\mu\right)^{m} h_{0}\left(a_{1}, a_{2}\right)
$$

for $h_{0}$ an arbitrary polynomial and $m>1$. After expansion in powers of $\mu$ we end 
up with a linear combination of hamiltonians in the plane, which we can associate with the free currents (4.1). So, from this point of view, the perturbed algebra of volume preserving diffeomorphisms arises upon perturbation of the free operators. In the matrix model we should take suitable $\mu$-dependent linear combinations of the operators in (3.22) in order to activate all the channels in (4.3). All this structure is strongly reminiscent of the similar phenomenon in interacting Liouville theory, where a vertex operator is expanded in a set of chiral components,

$$
e^{-\gamma j \phi(z, \bar{z})}=\sum_{m=-j}^{j} \xi_{m}^{j}(z)(-)^{j-m} \bar{\xi}_{m}^{j}(\bar{z})
$$

and the chiral "square roots" $\xi_{m}^{j}$ transform in an $S L(2, R)$ representation of spin $j$.

\section{Conclusions}

We have discussed several recent conjectures about the effect of the Liouville interaction on the $c=1$ model coupled to two dimensional quantum gravity. In particular, we find that Witten's ground cone is certainly deformed into a 3dimensional hyperboloid, at least in correlation functions. Similar evidence for the conjectured deformation of the Fermi surface is also found. It seems that the essential ingredient making possible the alluded deformations is the lack of chiral factorization in the Liouville sector when the cosmological constant is turned on. This is implied in the calculations on the cone by the point-spliting regularization used, and we propose that the conformal operators implementing the volume preserving diffeomorphisms of the quadric should be constructed as perturbations of the free fields appearing in the free case. It would be most interesting to make contact with exact treatments of the Liouville field which already incorporate this fact, like [14] [15]. One would like to see the algebraic structure encoded in the $\mu \neq 0$ hyperboloid as a sort of Backlund transform of the free cone. 
Regarding the moduli current algebra, we explicitly calculate the structure constants for non-zero cosmological constant, although serious regularization ambiguities are found in the Liouville sector. In spite of their simple form, we don't find any geometrical interpretation, which would be important in order to conjecture string field actions. As a matter of fact, certain combinations of $\mathrm{Z}$ operators do satisfy a volume preserving algebra under free OPE. This is because $\mathrm{Z}$ operators correspond to the hamiltonians generating volume preserving vector fields ( $\mathrm{J}$ operators). Free field OPE of the Z's corresponds to Poisson brackets squared of the hamiltonians, which in turn are equivalent to the conmutator of vector fields. So, if the picture advocated in the previous section is correct, there are certain $\mu$-dependent deformations which under free OPE, enjoy the J's algebra squared.

. Acknowledgements. This work arose from conversations with L. AlvarezGaumé, to whom I am indebted for his patient tutoring and constant encouragement. I also thank César Gómez, Germán Sierra and Javier Mas for stimulating discussions, and the Cern-TH division for hospitality. This work has been supported by an FPI-MEC grant.

\section{Appendix 1}

In this appendix we give an explicit formula for (3.13) :

$$
I\left(s_{1}, s_{2}, s_{3}\right)=\prod_{j=1}^{s+1} \oint \frac{d x_{j}}{2 \pi i} x_{j}^{-2 s_{1}}\left(1+x_{j}\right)^{-2 s_{2}} \prod_{j<k}^{s+1}\left(x_{j}-x_{k}\right)^{2}
$$

the contour integrals are nested around 0 in the usual positive sense (counterclockwise). Of course, direct evaluation of the poles seems hopeless, because of the presence of the Vandermonde determinant squared in the integrand, which complicates the power expansion. We will use analitic continuation in the exponents. 
Consider the integral

$$
I_{\epsilon}(\alpha, \beta ; s)=\prod_{j=1}^{s+1} \int_{\gamma_{j}(\epsilon)} \frac{d x_{j}}{2 \pi i} x_{j}^{\alpha}\left(x_{j}-1\right)^{\beta} \prod_{j<k}^{s+1}\left(x_{j}-x_{k}\right)^{2}
$$

The contours $\gamma_{j}(\epsilon)$ are homotopic to the ones in (6.1)and defined as $D_{j}(\epsilon) \circ C_{j}(\epsilon)$, where $D_{j}(\epsilon)$ denotes a small clockwise circle of radius $\epsilon$ around 0 , and $C_{j}(\epsilon)=$ $[\epsilon+i 0 \rightarrow 1-\epsilon+i 0] \circ[1-\epsilon-i 0 \rightarrow \epsilon-i 0]$.

For $\alpha=-2 s_{2}$ and $\beta=-2 s_{1}$ we recover (6.1) for any value of $\epsilon$. This is because it converges at $x_{j}=\infty$, so that a change in the orientation of the contour can be traded by the shift $x_{j} \rightarrow x_{j}-1$. Now we evaluate (6.2) in the region of the $\alpha, \beta$ plane were it is convergent at 0 and 1 . This corresponds to $\alpha, \beta>-1$. So in this region we can evaluate the $\epsilon \rightarrow 0$ limit by simply neglecting the small $\epsilon$-circles $D_{j}(\epsilon)$, i.e:

$$
I(\alpha, \beta ; s) \equiv \lim _{\epsilon \rightarrow 0} I_{\epsilon}(\alpha, \beta ; s)=\prod_{j=1}^{s+1} \int_{C_{j}} \frac{d x_{j}}{2 \pi i} x_{j}^{\alpha}\left(x_{j}-1\right)^{\beta} \prod_{j<k}^{s+1}\left(x_{j}-x_{k}\right)^{2}
$$

where the contours $C_{j}$ are just the $\epsilon \rightarrow 0$ limit of $C_{j}(\epsilon)$. Now by simple evaluation of the monodromy:

$$
I(\alpha, \beta ; s)=\pi^{-s-1} e^{i \pi s \alpha}(-)^{s+1}(\sin \pi \alpha)^{s+1} \prod_{j=1}^{s+1} \int_{0}^{1} d x_{j} x_{j}^{\alpha}\left(x_{j}-1\right)^{\beta} \prod_{j<k}^{s+1}\left(x_{j}-x_{k}\right)^{2}
$$

The integral in the right hand side is the so called Selberg-Fattev-Dotsenko integral [9]. After evaluating it and using $\Gamma(x) \Gamma(1-x)=\frac{\pi}{\sin \pi x}$ we arrive at

$$
(-)^{\frac{(s+1)(s+2)}{2}} \pi^{s+1}(\sin \pi \alpha)^{-s-1}\left(\frac{\sin \pi(\alpha+\beta)}{\sin \pi \beta}\right)^{s+1} \prod_{j=1}^{s+1} j ! \prod_{j=0}^{s} \frac{\Gamma(-\alpha-\beta-s-1-j)}{\Gamma(-\alpha-j) \Gamma(-\beta-j)}
$$

which can be combined with (6.4) and analitically continued back to $\alpha=-2 s_{2}$ 
and $\beta=-2 s_{1}$. Finally:

$$
I\left(s_{1}, s_{2}, s_{3}\right) \equiv I\left(s_{1}, s_{2} ; s\right)=(-)^{\frac{s(s+1)}{2}} \prod_{j=1}^{s+1} j ! \prod_{j=0}^{s} \frac{\left(2 s_{1}+2 s_{2}-s-2-j\right) !}{\left(2 s_{1}-1-j\right) !\left(2 s_{2}-1-j\right) !}
$$




\section{REFERENCES}

1. D.J. Gross, I.R. Klebanov, and M.J. Newman: Nucl.Phys. 350 (1991) 621

2. A.M. Polyakov: Mod. Phys. Lett. A6(1991) 635

3. B.H. Lian and G.J. Zuckermann: Phys. Lett. 226(1991) 21. P. Bouwknegt, J.McCarthy and K. Pilch: CERN-TH. 6192/91

4. E. Witten: IASSNS-HEP-91/51

5. I.R. Klebanov and A.M. Polyakov: Mod. Phys. Lett. A6 (1991) 3273

6. G. Moore and N. Seiberg: Rutgers and Yale preprint, RU-91-29. YCTP-P1991. J. Avan and A. Jevicki: Brown preprints BROWN-HET-824 and 839. D. Minic, J. Polchinski and Z. Yang: Texas preprint UTTG-16-91. S.Das, A. Dhar, G. Mandal and S. Wadia: IASSNS-HEP-91/52 and 91/72.

7. E. Bergshoeff, M.P Blencowe and K.S. Stelle: Comm. Math. Phys. 128 (1990) 213. C. Pope, L. Romans and X. Shen: Nucl. Phys. B339 (1990) 191.

8. N. Seiberg: Prog. Theor. Phys. Suppl. 102 (1990) 319.

9. V.S. Dotsenko and V.A. Fateev: Nucl. Phys. 251 (1985) 691

10. D. Kutasov, E. Martinec and N. Seiberg: Princeton and Rutgers preprints, PUPT-1293, Ru-91-49. I.R. Klebanov: Princeton preprint PUPT-1302

11. M. Li: Santa Barbara preprint, UCSBBTH- 91-47.

12. A.R. Edmonds: Princeton Univ. Press., Princeton N.J. (1957)

13. U.H. Danielsson: Princeton preprint, PUPT- 1301

14. J.L. Gervais: Int J. Mod. Phys.A6 No.16 (1991) 2805

15. E.Braaten, T. Curtright and C. Thorn: Ann Phys. 197 (1983) 365 